\title{
Aprendizagem e Letramento Digital de Alunos Adultos: 0 Caso do Curso Técnico em Informática a Distância do IFES
}

\author{
Gustavo de Oliveira Andrade*1, Dulce Márcia Cruz²
}

\begin{abstract}
1 Investigador do Programa de Pós-Graduação em Educação. Universidade Federal de Santa Catarina (UFSC) - Campus Universitário Reitor João David Ferreira Lima - 88040-900 - Florianópolis - Santa Catarina - Brasil. andrade.goliveira@gmail.com

2 Pesquisadora do Programa de Pós-Graduação em Educação. Universidade Federal de Santa Catarina (UFSC) - Campus Universitário Reitor João David Ferreira Lima - 88040-900 - Florianópolis - Santa Catarina - Brasil. dulce.marcia@gmail.com
\end{abstract}

\section{Resumo}

As mídias estão cada vez mais presentes no cotidiano dos estudantes, principalmente porque a maioria deles já nasceu nesse mundo tecnológico. No entanto, ainda pouco se conhece sobre o letramento digital dos alunos adultos dos cursos técnicos. Por essa razão, o objetivo principal desta pesquisa foi investigar a relevância das mídias e da cultura digital na visão dos alunos a distância do curso Técnico em Informática do Instituto Federal do Espírito Santo (IFES). Os dados coletados na pesquisa qualitativa mostraram que, apesar de serem ativos e autônomos, tendo seus próprios equipamentos para estudar e acessar rotineiramente as mídias na vida pessoal, não usufruem de todos os recursos da EAD e não utilizam na educação formal todos os recursos disponíveis na cultura digital, especialmente as tecnologias móveis e os jogos eletrônicos. Concluímos que eles poderiam ser mais desafiados pela escola para expandir as habilidades de aprendizagem adquiridas no contato com a cultura digital e pela experiência de estudar a distância, qualificando assim positivamente o letramento digital dos estudantes.

Palavras-chave: Mídia; Educação a distância; Letramento digital. 


\title{
Adults Learning and Digital Literacy: The Case of Distance Informatics Technical Course From IFES
}

\begin{abstract}
The media are increasingly present in the daily lives of students, mainly because most of them was born in this technological world. However, little is known about the digital literacy of adult students of technical courses. For this reason, the main objective of this research was to investigate the relevance of media and digital culture according to the point view of the distance education students of Technical Course in Computer Science from the Federal Institute of Espírito Santo (IFES). The data collected in qualitative research showed that students, despite being active and independent, using their own equipment to study and routinely access the media on personal life, they are not enjoying all the features of distance learning and they are not using in formal education all available resources in digital culture, particularly mobile technologies and electronic games. We concluded that they could be challenged by the school to expand the learning skills acquired in contact with the digital culture and with the experience of studying at distance, qualifying the digital literacy of students positively.
\end{abstract}

Keywords: Media; Distance education; Digital literacy. 


\section{Introdução}

A sociedade contemporânea possui um diferencial inovador devido a grandes invenções que ocasionaram verdadeiras transformações na vida e na forma dos seres humanos encararem o mundo e utilizarem-se do arsenal tecnológico em constante mudança. As tecnologias se apresentam como instrumentos que promovem grande agilidade e que diversificam as atividades executadas pelo homem. É visível o ganho de desempenho que trazem aos trabalhos na atualidade e que antes eram executados de forma manual. As tecnologias, especialmente as digitais, vêm transformando a vida das pessoas no final do século $\mathrm{XX}$, afetando as afinidades sociais e as formas de trabalhar, de se informar, de se formar, de se distrair, de consumir, de falar, de entrar em contato, de consultar, de decidir e de pensar (Perrenoud, 2005).

Pellanda (2005) salienta que estamos vivendo num período significativo na sociedade do conhecimento, na qual o processo de informatização demanda que as pessoas desenvolvam a capacidade para habituar-se às transformações que vêm ocorrendo. As tecnologias permitem captar várias formas de mostrar o mesmo objeto, representando-o sob ângulos e meios diferentes: pelos movimentos, cenários, sons, integrando o racional e o afetivo, o dedutivo e o indutivo, o espaço e o tempo, o concreto e o abstrato (Moran, 2007).

Nesse contexto, a educação vem passando por mudanças em sua estrutura e função com o uso das mídias que abrem novas possibilidades para o trabalho do professor. Isso porque as tecnologias são pontes que abrem a sala de aula para o mundo, que representam, medeiam o nosso conhecimento do mundo. Mas nem todos têm acesso igual ou conseguem se apropriar do potencial das TIC (Tecnologias da Informação e Comunicação) do mesmo modo e com a mesma intensidade.

Estudos, tais como os de Lima, 2013; Moreira, Latini 2008; Hempe, Franciscato, 2011, mostram que a utilização dos recursos midiáticos na sala de aula pode agregar valores na assimilação de conteúdos propostos pelos professores.

No entanto, apesar das inúmeras experiências de inclusão das mídias que vêm sendo feitas na educação de modo geral, ainda pouco se conhece sobre sua relevância e presença no letramento digital dos alunos do ensino médio dos cursos técnicos. Nesse sentido, o objetivo principal deste trabalho foi o de investigar a relevância e presença das mídias no letramento digital dos alunos a distância do curso Técnico em Informática do Instituto Federal do Espírito Santo (IFES). Mais especificamente, 
buscou-se identificar e mensurar a importância das mídias na percepção dos alunos durante o processo de ensino-aprendizagem e quais são utilizadas por eles a partir da identificação do seu perfil midiático e do processo de letramento digital vivido.

\section{Tecnologias da Informação e Comunicação}

As facilidades de comunicação que a tecnologia oferece, tais como busca da informação, interatividade e conforto, vêm facilitando e sendo difundidas na sociedade. Junto com essas facilidades, Garcez (2007) afirma que a tecnologia é vista como a expressão material de um processo que se manifesta por meio de instrumentos e máquinas e cuja finalidade é melhorar a vida humana. Moran avalia que

(...) muitas formas de ensinar hoje não se justificam mais. Perdemos tempo demais, aprendemos muito pouco, desmotivamo-nos continuamente. Tanto professores como alunos têm a clara sensação de que muitas aulas convencionais estão ultrapassadas. (2003, p.11)

Hawkins (1995) apud Cruz (2001) destaca que:

Muitas experiências de aprendizagem de novas tecnologias em ambientes escolares simplesmente fracassaram, apesar das boas intenções. O problema é que, como algumas experiências têm mostrado, para que haja uma boa aprendizagem do professor para o uso das mídias em sala de aula, devem ser preenchidas algumas condições mínimas. É preciso que seja criada uma experiência concentrada para o docente enquanto está experimentando as novas práticas na sala de aula. Essa aprendizagem tem que se dar dentro de uma estrutura de apoio que possibilite uma resposta à necessidade do professor de estar envolvido em diálogo com seus pares e com pessoas que estejam experimentando as mesmas técnicas e tecnologias. Além disso, os professores devem aprender noções básicas sobre mídia, o que os ajudará a pensar, com uma nova perspectiva, sobre seu conteúdo e sua utilidade (2001, p. 51$52)$.

A referência a recursos tecnológicos na educação costuma remeter à televisão, ao telefone e, principalmente, ao computador. Mas, em se tratando de educação, qualquer meio de comunicação que expande a ação do professor é uma ferramenta tecnológica na busca da qualidade do processo de ensino-aprendizagem. Exemplos disso são o quadro negro e o giz, as duas ferramentas mais antigas e mais usadas na sala de aula. (Damasceno, 2010). 
As mídias possibilitam uma ampliação circunstancial de possibilidades de manuseio pelo professor dentro e fora da sala de aula. Cruz (2001) define mídia como "meio" de organizar e promover a informação.

Mídia é um termo originado do latim media (que quer dizer meios) e que é plural de medium. Abrasileirada, a palavra acabou sendo adotada no Brasil, podendo referir-se indistintamente tanto a uma ferramenta técnica (a internet), a um veículo em especial (a TV, por exemplo), a vários veículos (as mídias impressas) ou ao total dos meios como um conjunto ("a" mídia). Tradicionalmente, mídia é a abreviação do que também se costuma chamar de meios de comunicação ou mass media.

No plural abrasileirado, as mídias envolvem toda uma gama de tecnologias concebidas para permitir o acesso, o processamento, a transmissão de informação. Os níveis de conhecimento da sociedade são substancialmente aprimorados pelas mídias. O desenvolvimento, a difusão e o uso das mídias digitais constituem as forças condutoras das sociedades de informação e de conhecimento. Muitos países têm tirado maior partido da difusão das mídias, permitindo mais crescimento das suas economias.

Nesse segmento de melhora tanto pessoal como profissional na aquisição de conhecimento, as mídias vêm mudando a vida de muitas pessoas, por ser uma ferramenta agregada à vida cotidiana de cada um.

No campo da educação, Moran (2000, p. 63) ressalta que:

Ensinar com as novas mídias será uma revolução se mudarmos
simultaneamente os paradigmas convencionais do ensino, que mantêm
distantes professores e alunos. Caso contrário, conseguiremos dar um
verniz de modernidade, sem mexer no essencial. A internet é um novo
meio de comunicação, ainda incipiente, mas que pode ajudar-nos a
rever, a ampliar e a modificar muitas das formas atuais de ensinar e de
aprender.

As mídias têm funcionado como instrumentos de socialização das novas gerações por diversas formas de assimilação e apropriação de conteúdos e informações na vida cotidiana. Por esses fatores, podem ser consideradas como instrumentos ou dispositivos de produção e reprodução da indústria cultural mundializada.

\subsection{Letramento Digital dos alunos diante das novas mídias}


O termo letramento surgiu em meados dos anos 1980. É originário da palavra em inglês literacy que, traduzida para o português, recebeu o significado de letramento. A palavra passa a existir a partir da necessidade da ocorrência de novos fatos, novas ideias e novas maneiras de compreender os fenômenos da escrita no mundo social (Soares, 2006). Melo (2012) define que letramento é uma competência que adquirem aqueles que já se apropriaram do código escrito, já são alfabetizados e sabem usufruir da capacidade obtida em situações e circunstâncias diversas. Segundo Mey (1998), a relevância do letramento, tanto do tipo usual quanto do digital, vai muito além de se afirmar que é uma tecnologia de informação adquirida ativa ou passivamente. Enfatiza, também, que é muito mais do que saber ler e escrever ou navegar na internet.

O letramento digital consiste em saber utilizar os recursos para aplicá-los no cotidiano em benefício do próprio usuário. Precisa-se, nesse caso, indagar o porquê de se fazer uma busca na web, ou seja, saber qual a finalidade dessa informação para a vida a fim de promover a aquisição de um (novo) conhecimento (Moreira, 2012). O conceito pode ser definido não apenas como habilidade individual de ler e escrever ao ter acesso às mídias, mas pelas consequências que esse processo tem tanto no nível social como individual tal como é descrito por Silva apud Warschauer (2006, p. 2):

Letramento digital inclui uma combinação de equipamentos, conteúdo, habilidades, entendimento e apoio social, fazendo com que o usuário envolva-se em práticas sociais significativas. Segundo ele, o letramento e a educação afetam o acesso on-line no macronível, pois realizados em massa servem para acelerar o desenvolvimento econômico, criando uma "tecnologização" maior da sociedade. Por outro lado, também interferem no micronível individual, já que as habilidades de leitura, escrita e pensamento continuam sendo decisivas para a capacidade de utilização da internet.

Também entendido como alfabetização digital por alguns autores, o letramento digital está atrelado tanto à apropriação de uma tecnologia quanto ao aprendizado efetivo das práticas de escrita que circulam no ciberespaço.

Nesse sentido, Velloso (2010, p. 32) afirma que:

O processo de alfabetização que se desenvolve na interação do usuário com o computador, assim como o processo de alfabetização para a aprendizagem do sistema de escrita, tem uma especificidade técnica, sendo uma porta de entrada indispensável para a aquisição tanto do 
sistema de escrita alfabético quanto do sistema de códigos que regem um ambiente virtual.

Para que o aluno se torne um letrado digital, ele precisará assumir novos papéis, que vão além do simples manuseio do computador, para buscar um letramento midiático:

Não é possível ser letrado de forma absoluta, mas quanto maior o envolvimento do autor nos diversos gêneros, maior sua capacidade de aprendizagem e expressão nos diferentes gêneros digitais (inclusão/letramento). Dessa maneira, um letramento midiático seria um processo de síntese continuada e de aprendizagem constante (dado o processo tecnológico de inovação) das possibilidades das mídias digitais. Assim, um letramento midiático inclui as várias mídias e seus gêneros (numa relação de reciprocidade) em práticas sociais e enunciativas que podem ser vividas em diferentes graus de conhecimento, num processo constante e, pelo menos em tese, sem um fim definido. (CRUZ, 2013, p. 87).

Portanto, o uso das mídias, entendidas como novas ferramentas educacionais, requer novas metodologias, assim como, novas estratégias que façam com que os recursos disponibilizados pelos professores ganhem sentido em suas abordagens. Para isso, é necessário que o professor incorpore, em sua metodologia, novas formas e meios de apropriação dos recursos disponibilizados pelas tecnologias da informação e comunicação para que o processo educativo seja eficiente e aconteça de forma satisfatória. Para verificar de modo exploratório como vem se dando esse processo de letramento nos cursos técnicos é que foi feita a pesquisa que gerou uma monografia de conclusão de especialização que será descrita a seguir.

\section{Estudo de Caso}

Esta pesquisa foi desenvolvida junto aos alunos do curso Técnico em Informática na modalidade a distância do Instituto Federal do Espírito Santo (IFES). O currículo do curso Técnico em Informática do IFES foi baseado no trabalho realizado pelo Grupo de Pesquisa do Currículo de Referência Nacional (GPCRF MEC/UFSC-2010), segundo o qual o profissional técnico em informática deve possuir um determinado conjunto de competências e habilidades a fim de atingir o perfil profissional de conclusão do curso. O IFES possui um Centro de Ensino a Distância (CEAD) que é o principal responsável 
por todos os projetos e programas na área de Educação a Distância (EAD) da instituição. Atualmente conta 31 polos distribuídos em todo o estado do Espírito Santo. O curso Técnico em Informática, na modalidade a distância, faz parte da Rede e-Tec Brasil do Ministério da Educação, sendo vinculado ao Campus Colatina. O curso utilizase do ambiente virtual de ensino-aprendizagem (AVEA) - Moodle, uma plataforma com recursos que permitem produzir e gerenciar atividades educacionais baseadas na internet e/ou em redes locais.

Foram enviados, no período de 06/07/2013 a 30/07/2013, questionários para os 78 alunos matriculados nas disciplinas Banco de Dados e Protocolos e Serviços de Rede, representando uma amostra de 34\% em relação ao total de alunos matriculados em ambas as disciplinas. Obteve-se 27 respostas, $42 \%$ dos alunos ativos nas duas disciplinas que juntas somam 62 alunos.

A escolha pelos alunos dessas disciplinas deu-se pelo fato de estarem com quase $50 \%$ do curso concluído, contribuindo assim com uma boa verificação diante dos conhecimentos iniciais e dos adquiridos após a passagem por disciplinas básicas, em especial, a de Introdução a EAD, que, por sua vez, possui em sua ementa componentes essenciais para uma boa utilização dos recursos midiáticos e ferramentas básicas de comunicação e interação.

A proposta do questionário, intitulado Perfil do Aluno diante das Novas Tecnologias, foi a de buscar compreender como as mídias se fazem presentes na vida de cada aluno bem como a importância delas em sua vida profissional após o uso e manuseio desses novos recursos. O questionário foi dividido em quatro blocos com questões abertas e fechadas totalizando 30 perguntas ao todo. Os blocos foram divididos da seguinte maneira: Perfil (com intuito de conhecer o perfil inicial e socioeconômico do aluno); Caracterização (visando identificar seus costumes e manuseio diante das tecnologias); Efeitos gerados antes e após o curso Técnico em Informática do IFES (buscando os resultados obtidos em nível de conhecimento por uso das mídias antes e depois das disciplinas ofertadas no curso) e, por último, Comportamentos e Aprendizagem (cuja função foi analisar seus desempenhos e hábitos de aprendizagens por meio das mídias).

Para preservar a identidade dos alunos, na descrição e discussão dos resultados, não serão citados os nomes, mas serão identificados por Aluno A, Aluno B e assim por diante. 


\subsection{Descrição e análise dos resultados}

A pesquisa nos mostrou que numa parcela dos discentes do Curso Técnico em Informática, o perfil dos estudantes do IFES de cursos da EAD, que 52\% dos entrevistados são do sexo masculino e $48 \%$ do sexo feminino. Além dessa proporção quase simétrica entre homens e mulheres no curso técnico, outro fator que também chama atenção foi a idade dos interessados nessa modalidade de ensino, que apresentou uma média de 30 anos ou mais com $48 \%$ morando com cônjuge ou companheiro.

Esses estudantes têm renda per capita igual ou inferior a três salários mínimos e afirmaram frequentar outro curso na mesma modalidade a distância (26\%), enquanto $44 \%$ estão engajados em outro tipo de curso na modalidade presencial. Tais dados podem indicar que a cada ano cresce o interesse dos alunos em uma melhor qualificação por meio do uso das mídias através da popularização e aumento da oferta da educação a distância no país.

Dos pesquisados, $50 \%$ preferem acessar o ambiente virtual de ensinoaprendizagem do curso em seu próprio domicílio, e, apenas, 17\% utilizam os polos de apoio presencial para o acesso on-line, o que demonstra uma capacidade de acesso às mídias de modo individual e de âmbito doméstico.

Dos inúmeros recursos disponibilizados pela Web 2.0, as respostas mostraram que $19 \%$ dos acessos estão relacionados à pesquisa e leitura de notícias na rede, perfazendo em torno de cinco a oito horas semanais dedicadas à internet.

Quando questionados sobre a razão pela qual utilizam as mídias em seu dia a dia, os alunos responderam que elas favorecem sua atualização (30\%), a busca por novos desafios (20\%) e contribuem para melhorar a comunicação (18\%).

De forma sintética, os dados mostraram que a maioria acessa o ambiente virtual em sua própria casa, seguida do local de trabalho e do IFES, destacando-se o uso das mídias para leitura de notícias, pesquisas, acessos à caixa de e-mail, redes sociais além de atualização no dia a dia.

O planejamento do aluno na modalidade a distância requer um comprometimento na organização de seu tempo para se dedicar aos estudos e à pesquisa inerente às suas atividades do curso. Nesse sentido, 44\% estabelecem uma rotina diária ou semanal para confecção de suas atividades relacionadas aos estudos e $26 \%$ procuram estudar apenas em datas de provas e/ou entregas de trabalhos. 
As mídias estão presentes de algum modo nas mais diferentes formas na vida dos alunos. Os depoimentos dos alunos, descritos abaixo, reforçam essa percepção:

\footnotetext{
"As mídias são muito importantes para o desenvolvimento e aprendizado do curso, pois, é através delas que conseguimos estudar". (Aluno B)

"Essencial para agregar conhecimento ao que se propõe". (Aluno C)

"É importante, pois contribui e auxilia no processo de ensinoaprendizagem". (Aluno D)
}

O uso das mídias e dos recursos disponibilizados favorece o acesso à informação e comunicação a qualquer hora e lugar. Foi possível identificar que tanto no domicílio ou na Escola, os alunos do IFES, possuem à sua disposição recursos necessários para o seu processo de ensino nos mais diferentes meios de comunicação e acesso.

Sobre a importância e uso do computador no auxílio das atividades do dia a dia, os resultados apontam que $63 \%$ dos alunos indicaram que o computador possui um lugar de destaque para realização de suas tarefas, sendo que 56\% dos alunos utilizam a internet para expor suas observações e opiniões e 44\% visam o seu crescimento intelectual por meio das comunidades de práticas, fóruns e listas de discussão sobre a área de formação.

Já em relação às funções da internet, 70\% dos alunos responderam que utilizam a rede mundial de computadores para procurar informação, tais como endereço e telefone, enquanto 52\% para geração de trabalho, negócios ou renda.

É interessante notar como as mídias auxiliam nas tarefas diárias dos estudantes, e que as utilizam tanto para localização de informações como para a participação em fóruns, onde expõem observações e opiniões sobre variados assuntos, mostrando a variabilidade dos recursos midiáticos.

Em relação ao ambiente virtual do curso, a maioria dos respondentes afirmou possuir algum conhecimento e destacou que considera o recurso tarefa (presente no Moodle), 22\%, seguida de webconferência, 18\%, fórum, 15\%, e videoconferência como instrumentos essenciais para o seu aprendizado. Os dados revelam que $85 \%$ dos alunos indicaram que o uso das mídias os auxiliou no seu entendimento sobre determinado assunto nas disciplinas, ocasionando, assim, seu aprendizado.

Na última etapa do questionário, intitulada "Comportamentos e Aprendizagem", foi possível observar e constatar alguns aspectos inerentes às atitudes que os alunos do IFES utilizam para o seu processo de ensino-aprendizagem. A EaD apresenta várias 
vantagens que propiciam uma maior flexibilidade, eficácia e formação personalizada para cada aluno: 15\% consideram vantagem a EAD favorecer a autoaprendizagem, $22 \%$, a possibilidade de realizar o curso no seu próprio ritmo e $22 \%$, a diversificação dos locais de estudo. Por essas condições, a maioria (41\%) afirma que a flexibilidade é uma das principais vantagens da EAD.

Mais de $50 \%$ dos estudantes do IFES preferem estudar no período noturno, 59\% de modo individual em sua própria casa para realizarem outras atividades, como o trabalho no período diurno, tendo a possibilidade de realizar o curso no seu próprio ritmo e a diversificação dos locais de estudo (22\%). Esses dados mostram que a flexibilidade e a diversidade de locais de estudo são os pontos fortes da modalidade de ensino para os estudantes da amostra. Mas também mostram que a possibilidade de colaboração on-line ainda não é uma realidade dos alunos conectados.

Diante dos dados coletados no questionário, a maior parte dos alunos sempre utiliza os Pacotes Office (67\%), por auxiliar em seus trabalhos no dia a dia e também pela facilidade de uso e disponibilidade em quase todos os computadores nos mais diversos lugares como em casa, no trabalho e até mesmo no IFES.

Nesse sentido, a mobilidade é uma realidade entre os estudantes, pois $63 \%$ sempre salvam seus arquivos em dispositivos portáteis. Por outro lado, não é ainda através de tablets ou celulares que essa portabilidade acontece.

Dos estudantes, apenas 15\% utiliza sempre os tablets contra 59\% que nunca usam. Os celulares também são pouco utilizados para acessar o ambiente virtual, pois $59 \%$ não usam ou o fazem raramente.

No entanto, já existe uma parcela dos estudantes motivada e acostumada com isso, pois 26\% afirmaram quase sempre e $11 \%$ sempre (num total de $37 \%$ ) acessar dessa maneira. Mas essa mobilidade não se encontra na relação com os jogos, pois a maioria (82\%) dos entrevistados raramente ou nunca utiliza esse recurso para jogar pela internet, o que pode ser motivado pela faixa etária do grupo estudado.

\section{Conclusão}

O presente trabalho mostrou que os estudantes do curso Técnico em Informática do IFES participantes da amostra são mais velhos do que a média dos cursos presenciais, envolvidos em relacionamentos duradouros e distribuídos quase que de forma homogênea entre os dois sexos. 
Aproveitam a flexibilidade da EAD para realizar ao mesmo tempo outros cursos presenciais e a distância e utilizam seus próprios equipamentos para seus estudos, não dependendo das instituições para isso, o que coincide com o aumento das vendas de computadores, notebooks e celulares nos últimos anos no país.

Essa popularização mostra um processo de letramento digital em andamento, pois essa capacidade de acesso às mídias de modo individual e de âmbito doméstico está voltada à pesquisa e leitura de notícias na rede em uma carga horária constante com o objetivo de servir para atualização, busca por novos desafios e melhoria da comunicação.

O letramento digital aparece na habilidade de estabelecer uma rotina diária ou semanal para confecção de atividades estudantis e a indicação de uma maioria dos estudantes de que o computador possui um lugar de destaque para realização de suas tarefas.

A indicação dos hábitos de uso da internet mostrou uma crescente apropriação de seus recursos e compreensão da sua utilidade através da exposição de opiniões e da participação ativa para o crescimento intelectual em comunidades de práticas, fóruns e listas de discussão sobre a área de formação. Uma significativa parcela dos estudantes respondeu que utiliza a rede mundial de computadores para procurar informação e geração de trabalho, negócios ou renda.

Apesar da quase totalidade dos estudantes indicar que o uso das mídias os auxiliou no seu entendimento sobre determinado assunto nas disciplinas, ocasionando, assim, seu aprendizado, é possível entender que essa afirmação está relacionada à necessidade da tecnologia como meio básico da EAD. Pelos depoimentos citados acima, os estudantes demonstram essa percepção. No entanto, o letramento digital no espaço escolar também está relacionado ao uso que os professores fazem das mídias em forma de estratégias didáticas.

Nesse sentido, os estudantes apontaram que dentre as ferramentas presentes no ambiente virtual as que mais colaboraram para o aprendizado foram a tarefa, a webconferência (encontro virtual que possibilita o compartilhamento de apresentações, voz, textos e vídeos), videoconferência (encontro virtual que possibilita o contato visual e sonoro entre os participantes) e o fórum. Se considerarmos que o primeiro recurso, como o próprio nome indica, permite a publicação de tarefas, ou seja, de resultados de aprendizagem, podemos concluir que apenas as últimas, como ferramentas interativas (de imagem/som e texto) permitiram uma maior semelhança com as utilizadas no 
letramento vivido na internet de modo geral, a partir do diálogo, troca de informações e construção de conhecimento de forma colaborativa em grupo. Reafirma essa falta de sintonia do letramento digital quando acontece na escola o fato de os estudantes preferirem estudar individualmente, não aproveitando as possibilidades de diálogo que o ambiente virtual do curso proporciona.

Esses dados mostraram, ainda que de forma incipiente, que o estudante a distância dos cursos técnicos do IFES está se apropriando das mídias em seu cotidiano, mas ainda não conhece ou não utiliza alguns desses recursos (tecnologias móveis e jogos, por exemplo) que poderiam qualificar mais seu letramento digital. Também mostra que esse aluno adulto pode ser mais desafiado pela escola, de modo a estender suas habilidades dentro da educação formal, tendo em vista as habilidades já incorporadas em forma de atividades cotidianas de posse e uso das mídias. Se os professores pudessem perceber e responder a essas demandas, talvez o letramento midiático dos estudantes fosse acelerado e, ao mesmo tempo, qualificado.

A modalidade de ensino a distância possui elementos essenciais em sua concepção e forma de aplicabilidade. O aluno dessa modalidade prefere sua adesão por oferecer uma maior flexibilidade, eficácia e formação personalizada para cada aluno. O estudante da Era Digital se torna o sujeito da aprendizagem em todos os sentidos. Ele é o principal interessado no autodesenvolvimento e na sua avaliação. Ele se torna o centro, o eixo e o horizonte de todo o processo de educação. Esses alunos da atualidade são diferentes. Os professores precisam conhecer melhor o perfil do estudante com acesso a recursos digitais para dar início a uma nova etapa no processo de ensino-aprendizagem.

\section{Referências}

Cruz, D. M. (2001). O professor midiático: a formação docente para a educação a distância no ambiente virtual da videoconferência. Tese de Doutorado. Universidade Federal de Santa Catarina.

Cruz, D. M. (2013). Letramento midiático na educação a distância. In: Fidalgo, F. S. R.; Corradi, W. J.; Lima, R. N. de S.; Favacho, A.; Arruda, E. P.. (Org.). Educação a distância: meios, atores e processos. 1ed.Belo Horizonte: CAED UFMG. 
Damasceno, R. J. A. (2010). A Resistência do professor diante das Novas Tecnologias. Acesso em 28 de junho de 2013, disponível em http://meuartigo.brasilescola.com/educacao/a-resistencia-professor-diante-dasnovas-tecnologias.htm

Garcez, R. O. (2007). O uso da tecnologia de informação e comunicação, no ensino, por professores universitários. Dissertação de Mestrado. Universidade Federal de Pelotas.

Hawkins, J. O uso de novas tecnologias na educação. Revista TB. Rio de Janeiro: 120; 57-70, jan/mar, 1995.

Hempe, C., Franciscato, F. T., Midias no contexto Escolar: Investigação sobre o uso das Mídias na Sala de Aula presencial. XVI Seminário Interinstitucional de Ensino, Pesquisa e Extensão. UNICRUZ, 2011.

Mey, J. L. As vozes da sociedade: letramento, consciência e poder. Tradução de Maria da Glória de Morais. Tradução de: The voices of society: literacy, conscientiousness and power. DELTA, vol.14, n. 2, 1998.

Melo, N. M. de F. S. M. (2012). Práticas de Letramento Digital na formação de professores: avanços e limites do uso das mídias digitais na sala de aula. Acesso em 28 de junho de 2013, disponível em http:www.uniso.br/ead/hipertexto/anais/76 NiceiaMelo.pdf

Moran, J. M. Ensino-aprendizagem Inovadores com Tecnologias Audiovisuais e Telemáticas. In: Novas Tecnologias e Mediação Pedagógica. 6.ed. Campinas, SP: Papirus, 2000.

Moran, J. M., Masseto, M. e Behrens, M. Novas tecnologias e mediação pedagógica. 7. ed. Campinas: Papirus, 2003.

Moran, J. M. (2007). As mídias na educação. Acesso em 28 de junho de 2013, disponível em http://www.eca.usp.br/moran/midias educ.htm

Moreira, D. G., Latini, R. M., Recursos Midiáticos e Núcleo de Tecnologia Educacional: o que dizem os Professores de Química. XIV Encontro Nacional de Ensino de Química (XIV ENEQ). UFPR, 2008. 
Moreira, C. Letramento Digital: do conceito a prática. Anais do SIELP. Volume 2, Número 1. Uberlândia: EDUFU, 2012.

Lima, M. F., Formação dos professores para a inserção das mídias em sala de aula: uma proposta de ação, reflexão e transformação. HOLOS, Ano 29, Vol . 3, 2013.

Pellanda, N. M. C.; Schunzen, E. T. M. ; Schunzen Jr. K. Inclusão digital: tecendo redes afetivas / cognitivas. Rio de Janeiro: DP\&A, 2005.

Perrenoud, P. Escola e cidadania: o papel da escola na formação para a cidadania. Porto Alegre: Artmed, 2005.

Soares, M. Novas práticas de leitura e escrita: letramento na cibercultura. Educ. Soc. Campinas, vol. 23,n. 81, 2006.

Warschauer, M. Tecnologia e inclusão social: a exclusão digital em debate. São Paulo: Editora SENAC São Paulo, 2006.

Velloso, M. J. M. (2010). Letramento digital na escola: um estudo sobre a apropriação das interfaces da WEB 2.0. Dissertação de Mestrado. Pontifícia Universidade Católica de Minas Gerais. 